UNTTED STATES DEPARTMENT OF THE INTERIOR GEOLOGICAL SURVEY

\title{
PRINCIPAL FACTS FOR A GRAVITY SURVEY OF BALTAZOR KNOWN GEOTHERMAL RESOURCE AREA, NEVADA
} BY $\div$

Donald L. Peterson and Donald B. Hoover

\author{
Open-file Report 77-67C
}

1977

This report is preliminary and has not been edited or reviewed for conformity with U.S. Geological Survey standards and nomenclature. 


\section{DISCLAIMER}

This report was prepared as an account of work sponsored by an agency of the United States Government. Neither the United States Government nor any agency Thereof, nor any of their employees, makes any warranty, express or implied, or assumes any legal liability or responsibility for the accuracy, completeness, or usefulness of any information, apparatus, product, or process disclosed, or represents that its use would not infringe privately owned rights. Reference herein to any specific commercial product, process, or service by trade name, trademark, manufacturer, or otherwise does not necessarily constitute or imply its endorsement, recommendation, or favoring by the United States Government or any agency thereof. The views and opinions of authors expressed herein do not necessarily state or reflect those of the United States Government or any agency thereof. 


\section{DISCLAIMER}

Portions of this document may be illegible in electronic image products. Images are produced from the best available original document. 
PRINCIPAL FACTS FOR A GRAVITY SURVEY OF

BALTAZOR KNOWN GEOTHERMAL RESOURCE

AREA, NEVADA

\begin{abstract}
Explanation of the headings of the columns of the accompanying table of principal facts:
\end{abstract}

STATION IDENTIFICATION

LATITUDE

LONGITUDE

ELEV.

ST.

OBSERVED

THEORETICAL

TERRAIN

FREE AIR

BOUGUER

SPECIAL

BOUGUER ANOMALY
Gravity survey station number

North latitude in degrees, minutes, and hundredths of minutes.

West longitude in degrees, minutes, and hundredths of minutes.

Elevation in feet (to convert to meters, multiply by 0.3048 )

Non-applicable

Observed gravity in milligals.

Theoretical gravity in milligals computed using the "Geodetic Reference System 1967". Terrain correction in milligals computed for "Hammer Zones" "E" through "H". Free-air correction in milligals. Bouguer correction in milligals (includes elevation and curvature correction). Non-applicable Bouguer anomaly in milligals for an assumed density of 2.67 grams per cubic centimeter. 
SPEC. FIELD

Non-applicable

These data are referenced to a gravity base station in Denio, Nevada at the Post Office, 50 meters south of the Oregon-Nevada State line, 1.6 meters south of the door in the southeast corner of the concrete porch, near the mailbox. Site is monumented with a "USAF Gravity Station" disc (A.C.I.C. reference number 2352-1). Base value is 979945.94. 


\section{REFERENCES}

Geodetic Reference System 1967, International Assn. of Geodesy, Special Publication No. 3.

Hammer, Sigmund, 1939, Terrain corrections for gravimeter stations: Geophysics, v. 4, no. 3, p. 184-193. 
BALTAZOR KGRA NE. BASE STA DENJO NEV ACIC REF NO 2352

METER W177 MSV .4910

Density: 2.67 Meter 10: w-177 Date: 06/01/77

STATION

IDENTIFICATION

\begin{tabular}{|c|c|}
\hline $\begin{array}{l}\text { kgra } \\
\text { kgra } \\
\text { kgra } \\
\text { kqra } \\
\text { kgra }\end{array}$ & $\begin{array}{l}\text { DEO } 1 \\
\text { DEO } 2 \\
\text { DEO3 } \\
\text { DEO } 4 \\
\text { DEO5 }\end{array}$ \\
\hline $\begin{array}{l}\text { kgra } \\
\text { kqra } \\
\text { kgra } \\
\text { kgra } \\
\text { kgra }\end{array}$ & $\begin{array}{l}\text { DEO6 } \\
\text { DEO } \\
\text { DEO } \\
\text { DEO9 } \\
\text { DE } 10\end{array}$ \\
\hline $\begin{array}{l}\text { kgra } \\
\text { kgra } \\
\text { kgra } \\
\text { kgra } \\
\text { kgra }\end{array}$ & $\begin{array}{l}\text { DE } 11 \\
\text { DE } 12 \\
\text { DE } 13 \\
\text { DE } 14 \\
\text { DE } 15\end{array}$ \\
\hline $\begin{array}{l}\text { kgra } \\
\text { kgra } \\
\text { kgra } \\
\text { kgra } \\
\text { kgea }\end{array}$ & $\begin{array}{l}\text { DE } 16 \\
\text { DE } 17 \\
D E 18 \\
D E 19 \\
\text { DE } 20\end{array}$ \\
\hline $\begin{array}{l}\text { kgra } \\
\text { kgra } \\
\text { kgra } \\
\text { kgra } \\
\text { kgra }\end{array}$ & $\begin{array}{l}\text { DE2 } 1 \\
\text { DE2 } 2 \\
\text { DE2 } 3 \\
\text { DE } 24 \\
\text { DE } 25\end{array}$ \\
\hline $\begin{array}{l}\text { kgra } \\
\text { kgra } \\
\text { kgra } \\
\text { kgra } \\
\text { kgra }\end{array}$ & $\begin{array}{l}\text { DE26 } \\
\text { DE2 } 7 \\
\text { DE2 } \\
\text { DE29 } \\
\text { DE } 30\end{array}$ \\
\hline $\begin{array}{l}\text { kgra } \\
\text { kgra } \\
\text { kgra }\end{array}$ & $\begin{array}{l}\text { DE31 } \\
\text { DE32 } \\
\text { DE33 }\end{array}$ \\
\hline
\end{tabular}

LOCATIOK

LATITUDE LONGITUDE

4156.58

4155.62

4155.62

4154.58

4154.73

4153.84

4153.87

4153.03

4155.82

4155.82

4155.82

4154.95

4154.95

4153.88

4152.64

4] 52.10

4152.10

4152.12

4155.46

4155.31

4155.24

4155.13

4155.39

4154.99

4154.82

4154.68

4155.18

4155.08

4155.00

4156.68

4157.02
11840.92

11842.49

11842.90

11844.02

11844.08

11844.82

11844.09

11844.69

11044.29

11840.69

11841.18

11841.71

11840.70

11841.72

11842.28

11841.73

11842.90

11844.10

11842.70

11842.64

11842.56

11842.50

11842.59

11842.42

11842.68

11842.80

11843.00

11842.29

11842.15

11841.90

11841.72

11842.83

\section{GRAVITY}

4214.0

4234.0

4375.0

4215.0

4327.0

4751.0

4214.0

4219.0

4215.0

4288.0

4208.0

4208.0

4418.0

4266.0

4245.0

$4247.0^{\circ}$

4378.0

4214.0

4219.0

4248.1

4225.4

4211.0

4211.7

4215.6

4211.8

4212.4

4214.6

4189.0

4214.2

4215.6

4239.6

4303.0

5004.0
$979952.50-980341.51$

$979943.12-980341.51$

$979944.79-980339.95$

$979939.23-980340.17$

$979906.54-980338.84$

$979941.84-980338.88$

$979942.09-980337.63$

$979938.59-980337.58$

$979957.02-980341.80$

$979959.82-980341.80$

$979956.87-980341.80$

$979947.59-980340.50$

$979953.09-980340.50$

$979950.59-980338.90$

$979949-95-980337.04$

$979942.68-980336.23$

$979947.00-980336.23$

$979938.70-980336.26$

$979951.54-980341.37$

$979952.01-9 \varepsilon 0341.27$

$979951.32-980341.04$

$979951.13-980340.94$

$979950.83-980340.77$

$979951.73-980341.16$

$979949.77-980340.56$

$979948.41-980340.30$

$979947.00-980340.09$

$979952.38-980340.84$

$979953.77-980340.70$

$979953.63-980340.58$

$979953.09-960343.09$

$979910.32-980343.60$
CORRECTIONS

TERRAIN FREE-AIR BOUGUER SPECIAL

B DUGUER

SPEC.

0.26

0.69

2.09

1.91

396.22

398.10

$-145.03$

$-145.71$

$411.35-150.54$

$396.32-145.06$

$406.84-148.90$

0.00

0.00

0.00

0.00

0.00

3.20

0.26
0.28

0.28

1.07

446.69

$-163.42$

$396.22-145.03$

$396.69-145.20$

$396.32-145.06$

$403.18 \quad-147.56$

0.33

0.17

1.13

0.26

395.66

0.00

0.00

0.00

0.00

0.00

0.00

0.00

0.00

0.00

$415.40-144.82$

$401.11-146.81$

$399.14-146.09$

0.55

1.43

0.00

0.00

399.33

$411.64-150.64$

$396.22-145.03$

$396.69-145.20$

$399.43-146.19$

0.89

0.44

0.39

0.39
0.39

$397.29-145.42$

$395.94-144.92$

$396.01-144.95$

$396.37-145.08$

$396.02-144.95$

0.31

0.31

0.25

0.21

$396.07-144.97$

$396.28-145.05$

$393.87-144.17$

$396.24-145.03$

$396.37-145.08$

0.25

$398.63-145.90$

$404.59-148.07$

0.51

470.47

$-172.08$
0.00

0.00

0.00

0.00

0.00

0.00

0.00

0.00

0.00

0.00
0.00

0.00

0.00

0.00

0.00

0.00

0.00

0.00

0.00
$-130.69$

$-135.93$

$-135.49$

$-141.99$

$-139.84$

$-145.82$

$-145.58$

$-143.76$

$-147.70$

$-130.82$

$-133.93$

$-128.40$

-132.85
-134.82

$-133.38$

$-131.13$

$-138.03$

-146.07
-135.43

$-136.49$

$-138.26$

$-138 \cdot 36$

-138.26
-137.97

$-139.39$

$-140.35$

$-143.14$

$-137.05$

$-133.98$

$-132.99$

$-132.82$ 